\title{
Orthogonal Sets of Data Windows Constructed From Trigonometric Polynomials
}

\author{
C. A. Greenhall \\ Communications Systems Research Section
}

\begin{abstract}
This article gives suboptimal, easily computable substitutes for the discrete prolatespheroidal windows used by Thomson for spectral estimation. Trigonometric coefficients and energy leakages of the window polynomials are tabulated.
\end{abstract}

\section{Motivation}

In a paper [1] on precise time and time interval (PTTI) applications, D. Percival argued as follows for the increased use of frequency domain methods for studying PTTI data:

In contrast to common practise in many other physical sciences, the statistical analysis of PTTI data is often based directly on time domain techniques rather than on frequency domain (spectral analysis) techniques. The predominant analysis technique in the PTTI community, namely, the two-sample (or Allan) variance, is often used to indirectly infer frequency domain properties under the assumption of a power-law spectrum. Here we argue that direct use and estimation of the spectrum of PTTI data have a number of potential advantages. First, spectral estimators are typically scaled independent chisquare random variables with a known number of degrees of freedom. These properties allow easy computation of the variance of estimators of various quantities that are direct functions of the spectrum. Second, the effect of detrending data can be quantified more easily in the frequency domain than in the time domain. Third, the variance of estimators of the two-sample variance can be expressed in terms of readily estimated spectral density functions. This allows one to generate confidence intervals for the two-sample variance without explicitly assuming a statistical model. Fourth, there exist tractable statistical techniques for estimating the spectrum from data sampled on an unequally spaced grid or from data corrupted by a small proportion of additive outliers. The two-sample variance cannot be readily generalized to these situations.

In the course of testing frequency standards and distribution equipment for the Deep Space Network, the JPL Frequency Standards Laboratory generates computer files containing records of phase residuals of pairs of frequency sources. The statistical properties of these time series are routinely summarized by estimating the two-sample variance [2]. This article is a step toward the goal of supplementing this current ability with the ability to generate estimates of power spectral density of phase for Fourier frequencies $f$ below the Nyquist frequency of these records, typically $0.5 \mathrm{~Hz}$. These estimates, together with measurements of spectral density for higher $f$ provided by commercial real-time spectrum analyzers, could characterize the phase noise of frequency sources over many decades of $f$ extending from microhertz to kilohertz. Periodic disturbances, which are now detected haphazardly by visual 
inspection of the residuals or by noticing steps or oscillations in the plots of two-sample variance, could be detected unambigously by statistical procedures based on spectral estimators or periodograms.

In [1] quoted above, Percival recommends a new method of spectral estimation, due to Thomson [3], that is especially suited to situations in which the range of spectral densities to be estimated is large. The method uses multiple orthogonal data windows (also called weights or tapers), the approximate computation of which is the main subject here.

\section{The Thomson Spectral Estimation Method}

In the Thomson method for estimating the power spectrum of a stationary time series $x[n]$ given $N$ samples $x[0], \ldots$, $x[N-1]$, a frequency band $\left[f_{0}-W, f_{0}+W\right]$ is chosen, and an estimate for the spectral density value $S\left(f_{0}\right)$ is computed as an average of the windowed periodograms, namely,

$$
\widehat{S}\left(f_{0}\right)=\frac{1}{K} \sum_{k=0}^{K-1}\left|y_{k}\left(f_{0}\right)\right|^{2}
$$

where

$$
y_{k}(f)=\sum_{n=0}^{N-1} x[n] v_{k}[n ; N, W] e^{-i 2 \pi f n}
$$

The window sequences $v_{0}, \ldots, v_{N-1}$ are the discrete prolatespheroidal sequences (DPSS) of Slepian [4]. They are orthonormal and are leakage-optimal over the space of sequences index-limited to $0, \ldots, N-1$, in the sense that

(1) $v_{0}$ has the smallest leakage of all nonzero elements

(2) for $k>0, v_{k}$ has the smallest leakage of all nonzero elements orthogonal to $v_{0}, \ldots, v_{k-1}$

For a given bandwidth $W$, the leakage $L(g, W)$ of a function $g$ of discrete or continuous time is defined here as the fraction of its total energy contained in frequencies outside $[-W, W]$. The leakage $L\left(v_{k}, W\right)$ increases with $k$ and decreases with $W$. By virtue of the orthogonality of the $v_{k}$, the estimate of Eq. (1) has approximately $2 K$ degrees of freedom if $x$ is Gaussian, $S(f)$ is nearly constant for $\left|f-f_{0}\right| \leqslant W$, and the leakage of $\nu_{k-1}$ is small. Thus, by adjusting $W$ and $K$, one can achieve a tradeoff in Eq. (1) among variance, resolution, and the influence of frequencies outside $\left[f_{0}-w, f_{0}+W\right]$ (the usual meaning of "leakage").
Since the computation of the DPSS requires the solution of the eigensystem of an $N \times N$ matrix (or of an integral operator if $N$ is large), the design of easily computable suboptimal substitutes for them may be of value. In view of Nuttall's constructions of windows from cosine polynomials of low degree [5], one might expect trigonometric polynomials with both sines and cosines to make attractive materials for construction of DPSS substitutes. In fact, this idea has already been realized by Bronez ([6] , p. 1869) in his recent extension of the Thomson method to the more general situation of unevenly spaced and multidimensional data. The coefficients of his polynomials and their leakages are respectively the eigenvectors and eigenvalues of a certain matrix whose coefficients depend on $N$, the number of data. The aim of this article is to simplify the situation further for evenly spaced one-dimensional data by deriving the coefficients and leakages of an orthonormal set of continuous-time trigonometric polynomials that do not depend on $N$. They are converted to discrete-time data windows by sampling them at $N$ properly chosen points.

\section{Continuous-Time Windows}

In this article, $w$ is used to denote bandwidth in terms of the fundamental frequency unit, which is $1 / N$ for windows on $0, \ldots, N-1$, and 1 for windows on $[-1 / 2,1 / 2]$, as constructed below. It is assumed that $w$ is an integer (for the author's convenience only). Consider a time-limited trigonometric polynomial

$$
\begin{array}{rlrl}
\phi(x) & =\sum_{\nu=-M}^{M} c[\nu] e^{i 2 \pi \nu \mathrm{x}} & & |x| \leqslant 1 / 2 \\
& =0 & |x|>1 / 2
\end{array}
$$

of degree $\leqslant M$. Its Fourier transform is

$$
\Phi(y)=\sum_{\nu=-M}^{M} c[\nu] s(y-\nu)
$$

where

$$
s(y)=\frac{\sin \pi y}{\pi y}
$$

The polynomials sought can be defined immediately: their coefficient arrays are normalized eigenvectors of the positivedefinite matrix

$$
A[i, j]=\int_{|y|>w} s(y-i) s(y-j) d y, \quad i, j=-M \text { to } M
$$


and their leakages are the eigenvalues. Denote the resulting polynomials by $\phi_{k}(x ; w, M), k=0$ to $2 M$, and their coefficients by $c_{k}[\nu], \nu=-M$ to $M$, where the leakages $L\left(\phi_{k}, w\right)$ are taken in increasing order. The polynomials are orthonormal and leakage-optimal over the space of polynomials of degree $\leqslant M$. They are called trig prolates for short, because they can be regarded as finite-dimensional analogs of the prolate. spheroidal wave functions (PSWF) [7]. The symmetry of $A$ about its reverse diagonal forces the eigenfunctions to be either even or odd (the odd ones are multiplied by $\pm i$ to make them real), and empirically the above indexing gives $\phi_{k}$ the parity of $k$. The trig prolates share with the PSWF the property of double orthogonality: their transforms $\Phi_{k}(y ; w, M)$ are orthogonal over $[-w, w]$ as well as over $(-\infty, \infty)$.

The entries of $\boldsymbol{A}$ were computed as linear combinations of the integrals

$$
\int_{0}^{1}\left[\frac{\sin \pi y}{\pi(n+y)}\right]^{2} d y, \int_{0}^{1}\left[\frac{\sin ^{2} \pi y}{\pi^{2}(n+y)}\right] d y, n=0 \text { to } w+M-1
$$

which were computed by Romberg quadrature. The eigenvalues and eigenvectors were computed by EISPACK routines [8], [9]. Although the leakages decrease if $M$ increases, setting $M=w$ gives adequate performance (Section $V$ ).

Table 1 gives the coefficients and leakages of the trig prolates for $w=2$ to $5, M=w$, and for all $k$ such that $L\left(\phi_{k}, w\right)<$ 0.01 . Figure 1 shows the frequency response $\left|\Phi_{k}(y ; 4,4)\right|^{2}$ for $k=0$ and 4 . Comparing these with Thomson's graphs of the frequency responses of the DPSS for large $N$ ([3], Fig. 2), one can see that the maximum sidelobes of the trig prolates are at most $2.5 \mathrm{~dB}$ above those of the corresponding DPSS, although the sidelobe structure of the trig prolates is less regular.

\section{Discrete-Time Windows}

An orthogonal set of windows for data on $0, \ldots, N-1$ and bandwidth $W=w / N$ is constructed by sampling the $\phi_{k}$ as follows:

$$
\begin{array}{r}
u_{k}[n ; N, W, M]=\phi_{k}\left(\frac{n-(N-1) / 2}{N}, w, M\right), \\
n=0 \text { to } N-1, k=0 \text { to } 2 M
\end{array}
$$

Notice that the denominator is $N$ instead of $N-1$. This has two beneficial effects :

(1) the basis functions $e^{i 2 \pi v x}$ remain orthogonal when so sampled
(2) their discrete-time transforms are more closely related to their continuous-time transforms (see below)

The discrete-time windows $u_{k}$ are called sampled trig prolates. Orthogonality is preserved, namely,

$$
\sum_{n=0}^{N-1} u_{i}[n] u_{j}[n]=N \delta_{i j}
$$

Their discrete-time Fourier transforms are

$$
U_{k}(f ; N, W, M)=e^{-i \pi(N-1) f} \sum_{\nu=-M}^{M} c_{k}[\nu] s(N f-\nu ; N)
$$

where

$$
s(y ; N)=\frac{\sin \pi y}{\sin (\pi y / N)}
$$

(compare with Eqs. (3) and (4)). A spectral estimate of Thomson type is obtained by using $(1 / \sqrt{N}) u_{k}[n ; N, W, M]$ in place of $v_{k}[n ; N, W]$ in Eqs. (1) and (2).

\section{Comparison with Optimal Windows}

How much leakage performance is lost by the use of these suboptimal windows? Let $L\left(\phi_{k}, w\right), L\left(u_{k}, N, w\right)$, and 1 $\lambda_{k}(N, W)$, where $N W=w=M$, be the leakages of the trig prolates, the sampled trig prolates, and the optimal DPSS respectively. Evaluating $L\left(u_{k}\right)$ by means of the quadratic form in the numerator of Eq. (32) of [6], it is found that $L\left(u_{k}\right)$ is between $0 \mathrm{~dB}$ and $1.2 \mathrm{~dB}$ less than $L\left(\phi_{k}\right)$ for the instances of $w$ and $k$ given in Table 1 and for $N=8 w$. For $N=$ $16 \mathrm{w}$, replace $1.2 \mathrm{~dB}$ by $0.6 \mathrm{~dB}$. Thus, the sampled trig prolates have slightly less leakage than the trig prolates. Table 2 gives the ratio of sampled trig prolate leakage to DPSS leakage, which was computed by solving the eigensystem given by Eq. (2.9) of [3]. For $N=8 w$, the leakages of the trig prolates are $1.2 \mathrm{~dB}$ to $5.4 \mathrm{~dB}$ greater than those of the optimal DPSS; for $N=16 w$, the range is $1.2 \mathrm{~dB}$ to $2.6 \mathrm{~dB}$. The leakages of the corresponding Bronez discrete polynomial windows, which form the leakage-optimal set of discrete-time poly. nomials of degree $\leqslant M$, necessarily lie between those of the sampled trig prolates and those of the DPSS

\section{Conclusions}

This article has described several orthonormal systems of data windows, called the sampled trig prolates, that can be used in the Thomson method of spectral estimation. For $w=$ 
$N W=2$ to 5 , and $4 W$ not greater than the Nyquist frequency (i.e., $N \geqslant 16 w$ ), the user of these windows pays a leakage penalty of at most $2.6 \mathrm{~dB}$ for not using the optimal DPSS windows. In return, one merely needs to evaluate certain trigonometric polynomials of degree $w$, with coefficients given in Table 1 , at $N$ points according to $\mathrm{Eq}$. (5). By contrast, the evaluation of the DPSS windows requires the solution of an $N \times N$ symmetric Toeplitz matrix eigensystem. If
$N$ is large, one can proceed by solving a symmetric $J \times J$ eigensystem obtained from the approximation of a certain integral operator by Gaussian quadrature, in which the required number of knots $J$ depends on the details of floating-point hardware and mathematical software ([3], pp. 1090-1091). The prospective user of the Thomson method might regard the 2.6-dB penalty as an attractive tradeoff for avoiding these complexities.

\section{References}

[1] D. B. Percival, "A Reappraisal of Frequency Domain Techniques for Assessing Frequency Stability Measurements," Proc. 19th Precise Time and Time Interval Applications and Planning Meeting, U.S. Naval Observatory, Washington, D.C., 1987.

[2] C. A. Greenhall, "Frequency Stability Review," TDA Progress Report 42.88, vol. October-December 1986, Jet Propulsion Laboratory, Pasadena, California, pp. 200-212, February $15,1987$.

[3] D. J. Thomson, "Spectrum Estimation and Harmonic Analysis," Proc. IEEE, vol. 70, pp. 1055-1096, 1982.

[4] D. Slepian, "Prolate Spheroidal Wave Functions, Fourier Analysis, and Uncertainty V: The Discrete Case," Bell System Tech. J., vol. 57, pp. 1371-1430, 1978.

[5] A. H. Nuttall, "Some Windows With Very Good Sidelobe Behavior," IEEE Trans. on Acoustics, Speech, and Signal Processing, vol. ASSP-29, pp. 84-91, 1981.

[6] T. P. Bronez, "Spectral Estimation of Irregularly Sampled Multidimensional Processes by Generalized Prolate Spheroidal Sequences," IEEE Trans. on Acoustics, Speech, and Signal Processing, vol. 36, pp. 1862-1873, 1988.

[7] D. Slepian, "Some Comments on Fourier Analysis, Uncertainty, and Modeling," SIAM Review, vol. 25, pp. 379-393, 1983.

[8] B. T. Smith et al., Matrix Eigensystem Routines-EISPACK Guide, Lecture Notes in Computer Science 6, Berlin: Springer-Verlag, 1976.

[9] B. S. Garbow et al., Matrix Eigensystem Routines-EISPACK Guide Extension, Lecture Notes in Computer Science 51, Berlin: Springer-Verlag, 1977. 
Table 1. Sine-cosine coefficients $a_{k}[\nu]$ and leakage $L\left(\phi_{k}, w\right)$ for trig prolate $\phi_{k}(x ; w, w)=a_{k}[0]+2 \sum_{\nu=1}^{M} a_{k}[\nu] \cos 2 \pi \nu x(k$ oven)
\[ \text { or } 2 \sum_{\nu=1}^{M} a_{k}[\nu] \sin 2 \pi \nu x(k \text { odd }) \]

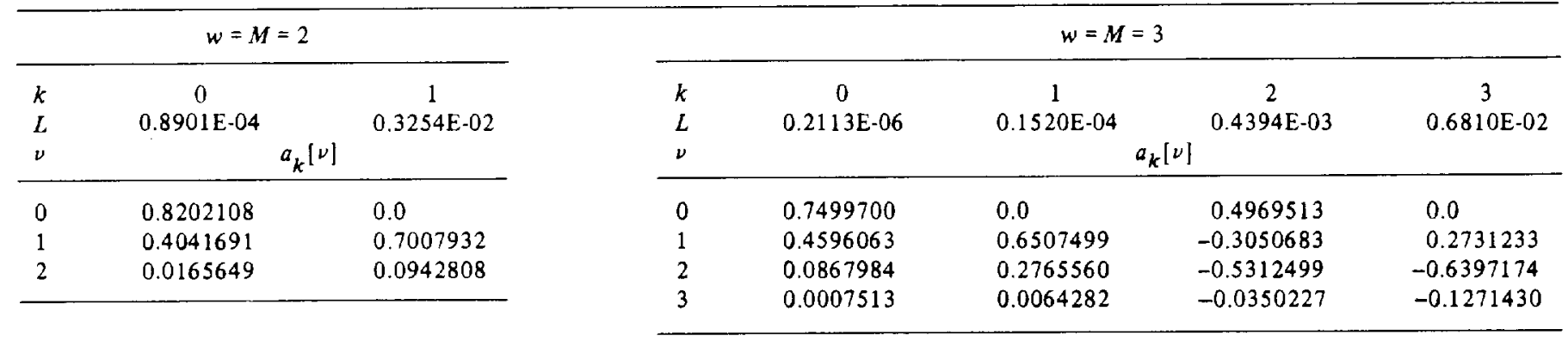

\begin{tabular}{|c|c|c|c|c|c|}
\hline \multicolumn{6}{|c|}{$w=M=4$} \\
\hline$k$ & 0 & 1 & 2 & 3 & 4 \\
\hline$L$ & $0.4376 \mathrm{E}-09$ & $0.4203 \mathrm{E}-07$ & $0.1965 \mathrm{E}-05$ & $0.5382 \mathrm{E}-04$ & $0.9029 \mathrm{E}-03$ \\
\hline$\nu$ & & & $a_{k}[\nu]$ & & \\
\hline 0 & 0.6996910 & 0.0 & 0.4783016 & 0.0 & -0.3862293 \\
\hline 1 & 0.4830013 & 0.5927723 & -0.1666510 & 0.3540569 & 0.3223025 \\
\hline 2 & 0.1473918 & 0.3805986 & -0.5724443 & -0.4929565 & -0.0856254 \\
\hline 3 & 0.0141997 & 0.0613650 & -0.1736202 & -0.3626279 & -0.5584413 \\
\hline 4 & 0.0000368 & 0.0003329 & -0.0022015 & -0.0117722 & -0.0484379 \\
\hline
\end{tabular}

\begin{tabular}{|c|c|c|c|c|c|c|c|}
\hline \multicolumn{8}{|c|}{$w=M=5$} \\
\hline$k$ & 0 & 1 & 2 & 3 & 4 & 5 & 6 \\
\hline 0 & 0.6632850 & 0.0 & 0.4560698 & 0.0 & -0.3821638 & 0.0 & 0.3246026 \\
\hline 1 & 0.4915713 & 0.5401300 & -0.0704481 & 0.3866087 & 0.2527019 & -0.2216043 & -0.2957322 \\
\hline 4 & 0.0019243 & 0.0105462 & -0.0379143 & -0.1037856 & -0.2286313 & -0.4072901 & -0.5631039 \\
\hline 5 & 0.0000018 & 0.0000191 & -0.0001319 & -0.0007467 & -0.0037712 & -0.0165910 & -0.0588589 \\
\hline
\end{tabular}

Table 2. Ratio (dB) of sampled trig prolate leakage to optimal DPSS leakagea

\begin{tabular}{lccccccc}
\hline $\begin{array}{l}k \\
w\end{array}$ & 0 & 1 & 2 & 3 & 4 & 5 & 6 \\
\hline 2 & $2.1,1.9$ & $1.2,1.2$ & & & & & \\
3 & $2.7,2.0$ & $2.4,2.2$ & $2.0,1.9$ & $1.3,1.3$ & & & \\
4 & $3.7,1.8$ & $2.8,1.7$ & $2.6,2.1$ & $2.4,2.2$ & $1.9,1.9$ & & \\
5 & $5.4,2.6$ & $4.2,2.1$ & $3.3,1.9$ & $2.7,2.0$ & $2.6,2.2$ & $2.4,2.2$ & $1.9,1.8$ \\
\hline \multicolumn{7}{l}{ a The first entry is for $N=8 w:$ the second entry is for $N=16 w}$. \\
\hline
\end{tabular}




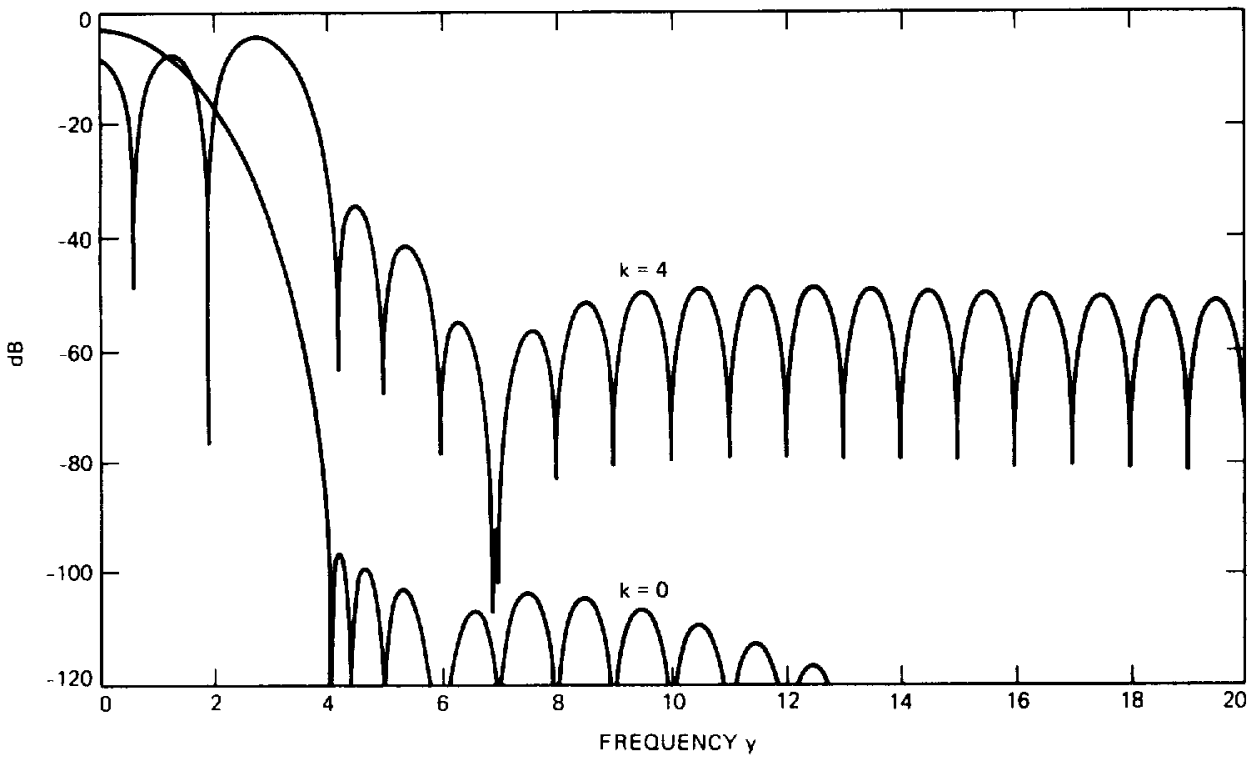

Fig. 1. Frequency response of trig prolate windows for bandwidth $w=4$, degree $M=4$. The total energy of each window is 1 . 


\title{
DSN 70-Meter Antenna Microwave Optics Design and Performance Improvements Part I: Design Optimization
}

\author{
D. A. Bathker \\ Ground Antenna and Facilities Engineering Section \\ S. D. Slobin \\ Telecommunications Systems Section
}

\begin{abstract}
This article reviews the design optimizations associated with the microwave and structural upgrade of the DSN 64-m antennas. Expected area efficiency/gain performances at $S$ - and $X$-band are given for both the original $64-\mathrm{m}$ systems and the upgraded 70-m systems, and error estimates are developed. The DSN 70-m Upgrade Project specifications, based on predesign estimates, were 1.4-dB gain at S-band and 1.9-dB at $X$-band, with no degradation to critical receiving system noise temperatures. The measurements show an $S$-band gain increase of $1.9 \mathrm{~dB}$ and an average increase of $2.1 \mathrm{~dB}$ at $X$-band. The Project also delivered small receiving system noise decreases at both frequency bands. The three DSN 70-m antennas, in the initial state of mechanical adjustment as of the end of calendar year 1988, are performing with very high peak microwave area efficiencies at very nearly the engineering design expectations of 76 percent at $S$-band and 71 percent at $X$-band.
\end{abstract}

\section{70-m Microwave Optics Design}

A 1975 engineering study identified several technical options for significantly increasing the microwave performance of the DSN 64-m antennas. ${ }^{1}$ Between 1975 and 1985, many of those optionswere implemented, providing improved X-band reception for the Voyager Jupiter, Saturn, and Uranus encounters. Those options included improved structural bracing of the main reflector to reduce the effects of gravity distortion,

\footnotetext{
${ }^{1}$ Radio Frequency Figure of Merit Enhancement Study, DSN 64-M Antennas, JPL 890-47 (internal document), Jet Propulsion Laboratory, Pasadena, California, June 1, 1975.
}

improved feedhorn design to obtain better aperture illumination, automated two-axis subreflector focusing for improved gain with elevation angle changes, and improved preamplifiers.

After these initial improvements, three options from the 1975 report remained unincorporated-increasing the physical diameter of the antenna, increasing the antenna aperture efficiency, and improving panel manufacturing and setting. Predesign work estimated that approximately $0.6 \mathrm{~dB}$ (15 percent) gain increase could be obtained from uniform illumination and an 0.8-dB (20 percent) increase could be obtained from the greater physical area (at $S$ - and X-bands). In addition, improved manufacturing and panel setting precisions for the individual main reflector panels and subreflector would provide an addi- 
tional $0.5-\mathrm{dB}$ (12-percent) improvement at X-band. Thus the 64-70-m Upgrade Project was established, with goals of 1.4-dB and 1.9. $\mathrm{dB}$ increases at $\mathrm{S}$ - and $\mathrm{X}$-band, respectively. To achieve $1.9-\mathrm{dB}$ gain increase over the $64-\mathrm{m}$ antenna by physical area alone would require a conventional $80-\mathrm{m}$ antenna. Alternatively, for the same performance level, the 64-m antenna could be augmented by arraying it with a conventional $47-m$ antenna.

The 64-m DSN antennas had conventionally profiled paraboloidal main reflectors and hyperboloidal subreflectors. These Cassegrainian systems were characterized by tapered aperture illumination intensity. It was expected that application of the modern dual-reflector contour shaping technique, which achieves uniform aperture illumination intensity and phase, could be successfully applied to the asymmetric feed geometry in place on the 64-m antennas. The asymmetric feed geometry provides multiple frequency feeding from a Cassegrain feed tricone. The tricone and associated rotatable subreflector effectively form a large, efficient bandswitch. For perfect main reflector axis pointing from such asymmetrically fed systems, a subreflector that is not a simple figure of revolution is required.

Designing the microwave optics for specially contoured surfaces begins with the selection of the feedhorn radiation pattern. A key design constraint was to retain the $64-\mathrm{m}$ antenna tricone subassembly, which includes the receivers and transmitters, the feedcone housings containing the feedhorns, associated microwave plumbing, and connections to the low-noise preamplifiers. By retaining this 44 -ft-high subassembly, standard DSN feedhorns critical to design and resulting microwave performances were economically retained. However, this constrained the microwave optics design in terms of the feedhorn beamwidth (which influences forward spillover based on subreflector diameter), and Cassegrain optics focal length (feed magnification factor). Despite this constraint, an excellent overall microwave optics design was obtained. The primary tradeoffs were to balance forward spillover against subreflector cost (a function of subreflector diameter), and selection of the rear spillover level past the main reflector rim (which influences critical reception noise). The design balance achieved about a 4-percent forward spillover (96-percent spillover efficiency). Although one might do somewhat better in an unconstrained design, the tricone retention and the savings in subreflector cost clearly established the acceptability of 96-percent forward spillover efficiency with standard feedhorns. In the future, slightly larger feedhorns might improve forward spillover efficiency by an additional 2 percent.

A very slight imbalance in illumination intensity results from the inherent asymmetric feed geometry. This amounts to about $2-\mathrm{dB}$ amplitude ramping, occurring near the main reflector edges, on a diameter including the asymmetric plane. However, the design is phase perfect; loss due to the slight intensity ramp is less than 0.5 percent at X-band.

The microwave optics design was optimized for X-band $(8420-\mathrm{MHz})$ area efficiency (gain) and noise, which constitute the $G / T$ receiving system performance index. This was done for an operating elevation angle of $30 \mathrm{deg}$ and a total receiving system noise level of 15 kelvins, since for nearly half of all tracking hours, the DSN antennas operate below 30-deg elevation. A total receiving system noise level of 15 kelvins at 30-deg elevation at X-band is an optimistic goal and difficult to achieve; the optimization is therefore conservatively biased in the low-noise direction. It should be pointed out that optimum G/T-balanced very-low-noise antennas sacrifice some gain in order to maintain very low rear spillover. Higher noise antennas, properly $\mathrm{G} / \mathrm{T}$ balanced for the higher attendant receiving noise, are capable of slightly higher gain, but do not achieve the overall better $G / T$ performance. A clear way to envision a very-low-noise optimized reflector antenna is to view the outer main reflector as intentionally not well illuminated, therefore not instrumental in developing overall system main-beam gain. Alternatively, in signal reception, the feed is purposefully not responsive to signal or noise from the rim regions and beyond; rather, the reflector edge region functions as an earth-noise shield, keeping the other critical factor (total system noise) controlled. This was accomplished in the $70-\mathrm{m}$ DSN design by synthesizing the dual-shaped surfaces for a 69-m (approximately) system, then adding a simple slopematched paraboloid noise-shield annulus.

At wavelengths shorter than X-band (e.g., 15-, 22- and future $32-\mathrm{GHz}$ ) the $70 \cdot \mathrm{m}$ DSN antennas will slowly tend, as a function of frequency increase, toward lower rear spillover noise, a consequence of the physics of subreflector edge diffraction. Conversely, at longer wavelengths (e.g., S-band) these antennas will unavoidably have slowly increasing rear spillover and attendant receiving noise.

Another consequence of increased longwave rear spillover arises when transmitting-a concern for transmit radiation power density safety. This issue was studied and it was concluded that in the environment of additional radiation sources beyond direct rear spillover, especially scatter due to the quadripod structure, the 70-m design, despite slightly increased S-band direct rear spillover, would not be a problem. The increased S-band direct spillover when transmitting remained numerically submerged below the scatter term, and radiation safety was not compromised. However, in the event that an ultra-low-noise S-band receiving system or an ultra-high-power S-band transmitting system is required on the $70-\mathrm{m}$ antennas, a fresh look at the adequacy of the X-band optimized optics 
operated at S-band will be necessary. In the event that additional main reflector annular rim shielding for $\mathbf{S}$-band is required, such an addition would be straightforward. Alternatively, a slightly larger S-band feedhorn or beamwidth-changing ellipsoidal reflector might suffice.

\section{64/70-m S-Band Design-Expected Performances}

Although the $70-\mathrm{m}$ microwave optics are X-band $\mathrm{G} / \mathrm{T}$ optimized, considerable effort was expended to predict $S$-band performance to meet the ongoing tracking requirements of a number of S-band deep-space spacecraft at remarkable and increasing distances. Tables 1 and 2 show the $S$-band design efficiency predictions for the $64 \cdot \mathrm{m}$ and $70-\mathrm{m}$ antennas, respectively. These predictions are the result of a consistent computational method. In Table 1 , the four-reflector S-band 64-m system is rigorously analyzed [1]. The analysis considers the $\mathrm{S}$-band feedhorn radiation pattern as scattered from the $\mathrm{S} / \mathrm{X}$ reflex-dichroic ellipsoidal and dichroic reflectors, and the conventional subreflector and main reflector. This analysis provides an efficiency subtotal as well as a pointing loss (squint). Pointing squint is an endemic effect in asymmetrically fed circular polarized antennas, and arises due to subtle cross-polarization field summations. The result is that the DSN antennas do not produce perfectly coaxial simultaneous $S / X$ beams. Rather, in the usual operating configuration, the $\mathrm{X}$-band beam peak is aimed as perfectly as possible and the simultaneous $\mathrm{S}$-band beam peak is unavoidably displaced a few millidegrees. Tables 1 and 2 account for this operating configuration by including a small S-band squint loss term. In Table 1 the illumination amplitude efficiency is, notably, 82 percent, representing the tapered illumination character. istics of paraboloid/hyperboloid RF optics. Other small terms in Table 1 lead to an overall $64-\mathrm{m}$ S-band prediction of 60.3 . percent aperture efficiency (61.51.dBi absolute gain) at $2285 \mathrm{MHz}$

In Table 2 , the $70-\mathrm{m} \mathrm{S}$-band performance increase due to dual-reflector shaping for uniform illumination is apparent in the illumination amplitude term - nearly 96 percent and fully $0.66 \mathrm{~dB}$ above the $64-\mathrm{m}$ in Table $1[2]$. Additional $70-\mathrm{m}$ improvements occur due to improved quadripod blockage and subreflector blocking terms. The subreflector is designed for reduced $\mathrm{X}$-band backscatter over the angular range subtending the Cassegrain feedccnes. At S-band, some backscatter improve. ment is realized. The $S$-band pointing squint effect is actually less for the shaped reflector $70-\mathrm{m}$ system than it was for the 64-m. Table 2 shows an overall $70-\mathrm{m}$ S-band prediction of 75.8-percent aperture efficiency $(63.28 \cdot \mathrm{dBi}$ absolute gain) at $2285 \mathrm{MHz}$. If the $\mathrm{S}$-band beam is peaked, in instances where simultaneous $\mathrm{S} / \mathrm{X}$ operation is not required, a small increase to 76.1 percent is predicted. The design-expected $64-70-\mathrm{m}$ Upgrade Project S-band gain performance increase is therefore $1.77 \mathrm{~dB}$, which is comfortably above the Project goal of $+1.4 \mathrm{~dB}$.

\section{64/70-m X-Band Design-Expected Performances}

A previous modification to the 64-m X-band system, employed for the Voyager Jupiter and subsequent encounters, was the dual-hybrid mode feedhorn [3]. At X-band, this modification provided $0.36 \mathrm{~dB}$ overall gain improvement over a conventional feed. Thus the 64-m X-band systems were already partially shaped by the use of the special, but somewhat narrowband dual hybrid mode feedhorn. In Table 3 this is reflected in the quite respectable 90-percent illumination amplitude term. Also in Table 3, the 64-m quadripod blockage term is treated the same as at S-band. Finally, Table 3 accepts the 64-m initial construction surface tolerances (main reflector panel manufacturing, setting, and hyperboloid manufacturing), even though 20 years have elapsed since the antennas were constructed, and with near certainty, the initial value $(1.14-\mathrm{mm}$ normal Ims) used in Table 3 is too low. The overall $64-\mathrm{m}$ $X$-band design-expected efficiency with initially specified surface tolerance remains high at 56.5 percent $(72.55-\mathrm{dBi}$ absolute gain) at $8420 \mathrm{MHz}$.

Table 4 gives the detailed $X$-band breakdown for the $70-\mathrm{m}$. Notably, the illumination amplitude efficiency is increased to a remarkable 98.2 percent when using an ordinary wideband single-hybrid mode (corrugated) feedhorn (JPL standard, 22.37-dBi gain). The 70-m subreflector blockage is improved over the $64-\mathrm{m}$, as is the quadripod blockage.

Additional and most valuable information became available for the $70-\mathrm{m}$ antennas based on $12-\mathrm{GHz}$ holographic imaging performed under contract by Eikontech, Ltd., of Sheffield, U.K. High-resolution aperture surface current (intensity) mapping revealed slightly wider quadripod shadowing than initially expected. An adjustment for $70 \cdot \mathrm{m}$ quadripod blockage based on holography is included in Table 4 as well as in Table 2. The adjustment combined with the original estimate still predicts a $70-\mathrm{m}$ quadripod efficiency improvement from $64-\mathrm{m}$ ( 85.6 percent to 90.1 percent), a result of considerable care with quadripod structural design. While it might be argued that the $12-\mathrm{GHz}$-based adjustment considered applicable at X-band should be further increased in the $70-\mathrm{m}$ $S$-band estimate due to wavelength, this has not been done in Table 2 due to lack of quantitative information. Lastly, in Table 4, an as-built adjustment for the stovepipe feed is made [4]. The stovepipe feed is a compromise design to avoid repackaging the $64 / 70-\mathrm{m} X$-band feedcones. Dual reflector 
shaping for uniform aperture illumination is not compatible with the previous 64-m dual-hybrid mode feedhorn. Rather, the JPL standard X-band feedhorn pattern $(22.37 \mathrm{dBi})$ was selected to synthesize the shaped reflector contours and to provide detailed performance estimates.

The stovepipe compromise allowed minimal mechanical changes within the three $\mathrm{X}$-band operational feedcones, but unfortunately results in a slightly greater forward spillover. The overall 70-m design-expected X-band efficiency is therefore 70.8 percent $(74.32-\mathrm{dBi}$ absolute gain) at $8420 \mathrm{MHz}$. Conceivably, the 70-m feedcones may eventually be upgraded, resulting in an increase in the (design potential) efficiency to 72.6 percent. A hypothetically perfect surface X-band $70-\mathrm{m}$ antenna with the design feedhorn would provide 76.5-percent efficiency, which is considered excellent for a very-low-noise centerline-symmetric main reflector antenna optics design with off set and dichroic feeding.

The design-expected 64-70-m Upgrade Project X-band gain performance increase is therefore $1.77 \mathrm{~dB}$, based on the originally specified $64-\mathrm{m}$ surface tolerance. The Project goal of $1.9 \mathrm{~dB}$ was based on earlier predesign estimates, which used a somewhat larger value for the 64-m surface tolerance.

\section{Accuracy of Design Expectations}

The procedure adopted herein is to identify what are believed to be the top four uncertainties in the predictions described above, and by calculations or judgment attempt to estimate high confidence limits (peaks). The peaks are first simply added, which is of course unduly conservative. It is argued here that one-third of such peaks may be considered the likely or approximate probable error.

The estimating processes (P.O. or physical optics and GTD or geometrical theory of diffraction) that cover the computed subtotals seen in Tables 1 through 4 are considered accurate to about one percent $( \pm 0.04 \mathrm{~dB})$ for P.O., and in this instance $\pm 0.10 \mathrm{~dB}$ for GTD, at X-band. An analytic surface description of the shaped subreflector profile is used in GTD and is known to be a poor fit (at the 1-percent efficiency level) at $\mathrm{X}$-band. The 64-m quadripod blockage efficiency is a poorly known factor. The likely performance is no better than the simple estimate, and could be $0.18 \mathrm{~dB}$ worse, based on recent $70 \cdot \mathrm{m}$ experience discussed below. The $70-\mathrm{m}$ quadripod is now better known due to holographic imaging, and the performance is not likely to be better than the simple estimate, and could be $0.07 \mathrm{~dB}$ worse.

Uncertainty about the effective surface tolerance, especially for the large main reflectors, not surprisingly makes the top four error list. For S-band this is not of significance, but the
$\mathrm{X}$-band estimating is very sensitive to surface tolerances. For the $64-\mathrm{m}$ the likely surface tolerance performance is certainly not better than that stated in Table 3 , and could be $0.3 \mathrm{~dB}$ worse in the DSS-14 case and $0.5 \mathrm{~dB}$ worse in the DSS-43/63 case, based on limited holographic imaging. Limited holographic imaging on one of the 64-m antennas indicates that 1.6to $1.7-\mathrm{mm} \mathrm{rms}$ (normal measure) was the likely condition just prior to decommissioning for the $70 \mathrm{~m}$ upgrade. For the $70-\mathrm{m}$ antennas there is fairly clear and repeatable holographic imagery to support the estimated effective $0.7-\mathrm{mm} \mathrm{rms} \mathrm{(normal)} \mathrm{at} \mathrm{all}$ three stations. Analysis of holographic imaging indicates that additional adjustment to $0.3 \mathrm{~mm}$ is possible as a theoretical limit; in practice, $0.4-\mathrm{mm} \mathrm{rms}$ may be achievable. It is considered that the $70 \mathrm{~m}$ surface performance at X-band cannot be better than quoted in Table 4 , and could conceivably be $0.2 \mathrm{~dB}$ worse. Finally, all four antenna/band combinations estimated in Tables 1-4 undoubtedly suffer unmodeled factors: imperfect static focus alignment at the rigging angle, small losses due to panel gaps, mechanical hysteresis, and many other details. Included in the top four error estimating elements is another \pm 1 percent $( \pm 0.04 \mathrm{~dB})$ to include at least some accounting for the many unmodeled small factors.

Table 5 summarizes results of the error estimating for DSN 64/70-m efficiency/gain design expectations. Both the conservative simple peak sums and the adopted likely onethird values are shown.

Taking the Table 5 low-end likely errors, Table 6 values are summarized as the design-expected (minimum-likely) area efficiencies to be expected from the various historic and present systems.

Tables 1 through 4 (design-expected) and Table 6 (designexpected minimum-likely) values need to be compared with field measured values. It is expected that the new $70-\mathrm{m}$ antennas may achieve essentially 75-percent area efficiency at S-band, and nearly 70-percent at X-band.

\section{Conclusion}

On the basis of a very careful and complete analysis of the numerous parameters involved in the makeup of the 64- and 70-meter antenna efficiencies, updated design estimates of gain and aperture efficiency have been developed at both $S$ and $\mathrm{X}$-band. The final predicted $\mathrm{S}$ - and $\mathrm{X}$-band gain improvements were both $1.77 \mathrm{~dB}$ (coincidentally), whereas the very early pre-Project goals (based on poor knowledge of "component" efficiencies) were $1.4 \mathrm{~dB}$ and $1.9 \mathrm{~dB}$, respectively. These design-gain improvements result from both larger antenna size $(0.78 \mathrm{~dB})$ and an increase of efficiency $(0.99 \mathrm{~dB})$, from 60 to 76 percent (S-band) and from 57 to 71 percent (X-band). 


\section{References}

[1] A. G. Cha, "Physical Optics Analysis of a Four-Reflector Antenna: Part I," TDA Progress Report 42-84, vol. October-December 1985. Jet Propulsion Laboratory, Pasadena, California, pp. 94-100, February 15, 1986.

[2] A. G. Cha, "Physical Optics Analysis of a Four-Reflector Antenna: Part II," TDA Progress Report 42-86, vol. April-June 1986, Jet Propulsion Laboratory, Pasadena, California, pp. 231-243, August 15, 1986.

[3] R. F. Thomas and D. A Bathker, "A Dual-Hybrid Mode Feedhorn for DSN Antenna Performance Enhancement," DSN Progress Report 42-22, Jet Propulsion Laboratory, Pasadena, California, pp. 101-108, August 15, 1974.

[4] F. Manshadi and R. Hartop, "Compound-Taper Feedhorn for the DSN 70-M Antennas," TDA Progress Report 42-90, vol. April-June 1987, Jet Propulsion Laboratory, Pasadena, California, pp. 81-88, August 15, 1987. 
Table 1. Design-expected 64-m S-band efficiency

\begin{tabular}{lll}
\hline \multicolumn{1}{c}{ Item } & Value & Notes \\
\hline $\begin{array}{l}\text { P.O. computed subtotal } \\
\quad \text { Includes: }\end{array}$ & 0.7752 & {$[1]$} \\
$\quad$ Rear spillover & & 0.998 \\
$\quad$ Forward spillover & & 0.970 \\
$\quad$ lllumination amplitude & & 0.824 \\
$\quad$ Illumination phase & & 0.984 \\
$\quad$ Cross polarization & & 0.999 \\
$\quad$ m $\neq$ 1 modes & 0.9616 & 0.980 \\
Central blockage & 0.8556 & \\
Quadripod blockage & 0.9993 & 6.25 percent, 1.2 opacity \\
Dichroic reflectivity & 0.998 & Relative to plane reflector \\
Surface reflectivities (4) & 0.9875 & Painted aluminum alloy \\
Surface tolerances (4) & 0.9897 & 1.26 mm normal (-0.055 dB) \\
Pointing squint & 0.9795 & X-band beam peaked \\
Waveguide dissipation & 0.9908 & \\
VSWR & 0.603 & $(61.51$ dBi at 2285 MHz) \\
TOTAL & & \\
\hline
\end{tabular}

Table 2. Design-expected 70-m S-band efficiency

\begin{tabular}{lll}
\multicolumn{1}{c}{ Item } & Value & \multicolumn{1}{c}{ Notes } \\
\hline $\begin{array}{l}\text { P.O. computed subtotal } \\
\quad \text { Includes: }\end{array}$ & 0.8925 & {$[2]$} \\
$\quad$ Rear spillover & & 0.994 \\
$\quad$ Forward spillover & & 0.959 \\
$\quad$ Illumination amplitude & & 0.959 \\
$\quad$ Illumination phase & & 0.994 \\
$\quad$ Cross polarization & & 1.00 \\
$\quad$ m $\neq 1$ modes & 0.983 & 0.980 \\
Central blockage & 0.9196 & \\
Quadripod blockage & 0.9795 & 3.42 percent, 1.2 opacity \\
$\quad$ Holography adjustment & 0.9993 & 5.1 percent effective \\
Dichroic reflectivity & 0.998 & Relative to plane reflector \\
Surface reflectivities (4) & 0.9946 & Painted aluminum alloy \\
Surface tolerances (4) & 0.996 & 0.86 mm normal (-0.024 dB) \\
Pointing squint & 0.9795 & X-band beam peaked \\
Waveguide dissipation & 0.9908 & \\
VSWR & 0.758 & $(63.28$ dBi at 2285 MHz) \\
TOTAL & & \\
\hline
\end{tabular}


Table 5. Estimated errors for 64/70-m design expectations

\begin{tabular}{lccc}
\hline \multicolumn{1}{c}{ Antenna/Band } & $\begin{array}{c}\text { Estimated } \\
\text { peak sum error, } \\
\mathrm{dB}\end{array}$ & $\begin{array}{c}\text { Estimated } \\
\text { likely error, } \\
\mathrm{dB}\end{array}$ & Reference \\
\hline 64-m/S-band & +0.08 & +0.03 & Table 1 \\
70-m/S-band & -0.26 & -0.09 & Table 2 \\
& +0.08 & +0.03 & Table 3 \\
64-m/X-band (DSS-14) & -0.15 & -0.05 & Table 3 \\
64-m/X-band (DSS-43/63) & +0.08 & +0.03 & Table 4 \\
& -0.56 & -0.19 & +0.03 \\
$70-\mathrm{m} / \mathrm{X}$-band & +0.08 & -0.25 & +0.05 \\
\end{tabular}

Table 6. Estimated minimum-likely efficiencies for 64/70-m systems

\begin{tabular}{llc}
\hline DSN system & Antenna & $\begin{array}{c}\text { Design-expected } \\
\text { (estimated minimum-likely) } \\
\text { efficiency }\end{array}$ \\
\hline 64-m S-band & All antennas & 0.591 \\
$70-\mathrm{m}$ S-band & All antennas & 0.749 \\
$64-\mathrm{m}$ X-band & DSS-14 & 0.541 \\
$64-\mathrm{m} \mathrm{X}$-band & DSS-43/63 & 0.533 \\
$70-\mathrm{m}$ X-band & All antennas & 0.686 \\
\hline
\end{tabular}


Table 5. Estimated errors for $64 / 70-m$ design expectations

\begin{tabular}{lccc}
\hline \multicolumn{1}{c}{ Antenna/Band } & $\begin{array}{c}\text { Estimated } \\
\text { peak sum error, } \\
\mathrm{dB}\end{array}$ & $\begin{array}{c}\text { Estimated } \\
\text { likely error, } \\
\mathrm{dB}\end{array}$ & Reference \\
\hline 64-m/S-band & +0.08 & +0.03 & Table 1 \\
$70-\mathrm{m} / \mathrm{S}$-band & -0.26 & -0.09 & Table 2 \\
& +0.08 & +0.03 & Table 3 \\
$64-\mathrm{m} / \mathrm{X}$-band (DSS-14) & -0.15 & -0.05 & Table 3 \\
& +0.08 & +0.03 & Table 4 \\
$64-\mathrm{m} / \mathrm{X}$-band (DSS-43/63) & -0.56 & -0.19 & +0.03 \\
& +0.08 & -0.25 & +0.05 \\
$70-\mathrm{m} / \mathrm{X}$-band & -0.76 & -0.14 & \\
\end{tabular}

Table 6. Estimated minimum-likely efticiencies for 64/70-m systems

\begin{tabular}{llc}
\hline DSN system & Antenna & $\begin{array}{c}\text { Design-expected } \\
\text { (estimated minimum-likely) } \\
\text { efficiency }\end{array}$ \\
\hline 64-m S-band & All antennas & 0.591 \\
70-m S-band & All antennas & 0.749 \\
64-m X-band & DSS-14 & 0.541 \\
64-m X-band & DSS-43/63 & 0.533 \\
70-m X-band & All antennas & 0.686 \\
\hline
\end{tabular}

